\title{
Switching Darunavir/Ritonavir to Darunavir/Cobicistat in Clinical Practice Experience with 150 patients through 48 weeks
}

\author{
de la Fuente $S^{*}$, Díaz A, Vicente N, and Ángel-Moreno A \\ Department of Internal Medicine, Hospital Universitario Puerta de Hierro-Majadahonda, Madrid, Spain
}

${ }^{*}$ Corresponding author: de la Fuente Moral S, PhD, Department of Internal Medicine, Hospital Universitario Puerta de Hierro-Majadahonda, Joaquín Rodrigo s/n, 28222, Majadahonda, Madrid, Spain, Fax: +34 91191 68 07, Tel: +34 9119163 42, E-mail: sarafm28@hotmail.com

Citation: de la Fuente S, Díaz A, Vicente N, Ángel-Moreno A (2018) Switching Darunavir/Ritonavir to Darunavir/ Cobicistat in Clinical practice - Experience with 150 patients through 48 weeks. J Aids Hiv Infec 4(1): 101. doi: 10.15744/2454-499X.4.101

Received Date: October 08, 2017 Accepted Date: February 26, 2018 Published Date: February 28, 2018

\begin{abstract}
Background: Darunavir (DRV) boosted with ritonavir (RTV) is currently among the preferred antiretroviral agents. Cobicistat (COBI), a new cytochrome P450 inhibitor is replacing RTV as pharmacoenhancer. Coformulated DRV/COBI has been approved based on bioequivalence data; however results on efficacy and safety in clinical practice are scarce. COBI inhibits creatinine tubular secretion, thus raising concerns about its renal safety.

The aim of this study was to evaluate the efficacy and safety of switching to DRV/COBI in virally suppressed patients.

Methods: We retrospectively studied first 150 virally suppressed patients while receiving DRV/RTV who switched to DRV/COBI. The primary end point was viral suppression at 48 weeks. Secondary outcomes included adverse events and changes in lipids and renal function.

Results: No adverse event were recorded. Out of 150 patients, 142 had available data at 24 weeks and 107 at 48 weeks. At this point 22 had changed their regimen, 15 by drug-drug interactions, 6 by simplification and one by dyslipidemia, and 9 lost their follow-up. At 48 weeks $97.2 \%$ maintained viral suppression, either if receiving triple, dual or monotherapy. An initial mild decrease in eGFR was observed $\left(6.19 \mathrm{ml} / \mathrm{min}\right.$; CI95\% 4.82-7.56, $\mathrm{p}<0.0001$ in patients with baseline eGFR $>70 \mathrm{ml} / \mathrm{min} / 1.73 \mathrm{~m}^{2}$, and $3.5 \mathrm{ml} / \mathrm{min}$; CI95\% 0.69$7.69, \mathrm{p}=0.04$ in those with baseline eGFR $50-70 \mathrm{ml} / \mathrm{min}$ ); then remained stable, without differences regardless of concomitant use of TDF. Triglycerides decreased slightly.

Conclusion: Trough 48 weeks, switching DRV/RTV to DRV/COBI is safe and maintains viral efficacy, without significant changes in lipid profile or renal function.

Keywords: ART simplification; DRV/COBI; ART boosting

List of abbreviations: AE: Adverse Events; ART: Antiretroviral Treatment; CI: Confidential Interval; CYP: Cytochrome P450; COBI: Cobicistat; eGFR: estimated glomerular filtration rate; DRV: Darunavir; HIV-1: type-1 human immunodeficiency virus; FTC: Eemtricitabine; INI: Integrase inhibitors; PI: Protease Inhibitor; RAMs: Resistance-Associated Mutations; RTV: Ritonavir; STR: SingleTablet Regimen; TDF: Tenofovir Disoproxil Fumarate; VF: Virologic Failure; VL: Viral Load
\end{abstract}

\section{Background}

Protease inhibitors (PIs) are potent antiretroviral agents, although their rapid intestinal and hepatic metabolism, mainly by the cytochrome P450 pathway, leads to a low systemic exposure. Boosting PIs has had a significant impact on the treatment of type-1 human immunodeficiency virus (HIV-1) infection by improving their pharmacokinetic profile, resulting in more durable efficacy, higher barrier to resistance, reduced pill burden and dosing frequency [1].

Low-dose ritonavir (RTV) has been widely used as pharmacokinetic enhancer, since it is a potent inhibitor of cytochrome P450 (CYP) 3A, thereby increasing the oral bioavailability of most HIV-1 PIs. Low-dose RTV may be associated with gastrointestinal disorders, hyperlipidemia, and clinically significant drug-drug interactions.

Darunavir boosted with RTV (DRV/r) has been widely used as part of antiretroviral treatment regimens (ART) in recent years. In two randomized, controlled Phase III trials, DRV/r 800/100 mg once daily demonstrated antiviral efficacy with long-term tolerability in treatment-naïve (ARTEMIS) [2] and treatment-experienced patients with no DRV resistance-associated mutations (RAMs) (ODIN) [3]. 
Cobicistat (COBI), a new selective inhibitor of CYP34A without antiretroviral effect, has become a new pharmacoenhancer of several drugs, including PIs and some integrase inhibitors (INI) of HIV-1 [4,5]. COBI weakly inhibits other cytochromes, like CYP2D6, and does not interfere with other drug metabolism pathways, such as glucuronidation, which could translate into a lower potential for pharmacological interactions [5]. Furthermore, COBI seems to present a better metabolic profile than RTV, with lower elevations in lipid levels in available studies [5]. On the other hand, COBI inhibits creatinine tubular secretion, increasing its serum levels without actual effect on the glomerular filtration rate.

COBI is a highly soluble molecule, which allows its co-formulation with other agents to reduce the pill burden [6]. DRV/COBI co-formulated in a single pill has been recently approved in Europe based on favorable bioequivalence data in phase I studies [7] and on expert opinion [8].

In naïve patients, once-daily COBI is a safe and effective pharmacoenhancer of atazanavir (ATV). COBI was noninferior to RTV in combination with ATV plus emtricitabine/tenofovir disoproxil fumarate (FTC/TDF), with high rates of viral success and comparable safety and tolerability profiles in an international, randomized, double-blind, double-dummy, active-controlled trial [9]. COBI was also studied as a component of a single-tablet regimen (STR) Stribild ${ }^{\circledR}$ (EVG/COBI/FTC/TDF), in 2 large randomized controlled trials that demonstrated its efficacy and safety [10,11]. Finally, DRV/COBI 800/150 mg once daily showed viral and immunologic responses similar to previous data for DRV/r 800/100 mg once daily in a 48 weeks Phase IIIb, open-label single-arm trial, mainly in treatment naïve patients [12].

However, data on safety and efficacy of DRV/COBI as a substitute for DRV/r in clinical practice are still scarce, and concerns about renal safety may be elicited, particularly if used along with nephrotoxic drugs or in patients with mild decreases of renal function.

In our institution, after the approval of co-formulated DRV/COBI, all patients on stable treatment with DRV/r once daily were directly switched to DRV/COBI once daily, if contraindications were not found by the treating doctor, including pregnancy or COBI-specific undesirable drug-drug interactions.

The aim of this study was to analyze the viral efficacy and safety, particularly renal and metabolic, of this switch.

\section{Material and Methods}

\section{Study Design and Study Population}

We conducted a retrospective study to evaluate the efficacy, safety and tolerability at 48 weeks of switching DRV/RTV to DRV/ COBI. We included all adult ( $>18$ years old) HIV-1 infected patients virally suppressed -viral load (VL) $<50$ copies $/ \mathrm{mL}$ - for more than 6 months on a stable regimen containing RTV-boosted DRV (800/100), who were switched to DRV/COBI (800/150), without changes in the other components of their regimen, between August 1, 2015 to February 29, 2016.

Patients were excluded if detectable viremia at switching, either because recent initiation of treatment $(n=16)$ or virological failure $(\mathrm{VF})(\mathrm{n}=24)$.

The study was approved by the local ethical committee.

\section{Study endpoints}

The primary end point was the proportion of patients who maintained viral suppression ( $\mathrm{VL}<50$ copies $/ \mathrm{mL})$ after switching and throughout 48 weeks. Secondary outcome measures included any treatment-emergent adverse events (AE), including those leading to discontinuation, and treatment change by any cause. Changes in renal function and lipid profile were also analyzed.

\section{Procedures}

Patient's basic demographics and clinical characteristics were recorded, as well as the rest of antiretroviral drugs included in their regimens.

HIV-1 viremia before switching was $<50 \mathrm{c} / \mathrm{dL}$ in all patients; we then recorded the first available VL after switching, and 24 and 48 weeks after. Serum levels of creatinine, total cholesterol, LDL-cholesterol, HDL-cholesterol and triglycerides were also recorded before and after switching to DRV/COBI, and 24 and 48 weeks after. The eGFR was calculated using the CKD-EPI formula. Finally, any adverse event potentially attributed to DRV/COBI had to be recorded, its severity and the eventual need of treatment change.

\section{Statistical Analysis}

The primary analysis included all virologic, clinical and laboratory data available after the patients had completed 48 weeks on treatment with DRV/COBI, or prematurely if they discontinued DRV/COBI by any reason. Safety assessments were summarized using descriptive statistics.

A p value $<0.05$ was considered as significant. All analyses were conducted using SPSS version 15.0 statistical software (SPSS Inc., Chicago, IL). 


\section{Results}

\section{Baseline Characteristics}

We included the first 150 patients switched from DRV/r to DRV/COBI. Demographic and general baseline characteristics are shown in Table 1.

\begin{tabular}{|c|c|}
\hline CHARACTERISTIC & $\mathrm{N}=150$ \\
\hline Age, y $($ mean $\pm S D)$ & $51.97 \pm 9.66$ \\
\hline Male (n, \%) & $120(80 \%)$ \\
\hline $\begin{array}{l}\text { Risk group (n, \%) } \\
\text { - MSM/BSX } \\
\text { - HTX } \\
\text { - IDU } \\
\text { - Transf/Vertical/Unknown }\end{array}$ & $\begin{array}{c}61(40.6 \%) \\
34(22.7 \%) \\
52(34.7 \%) \\
3(2 \%)\end{array}$ \\
\hline CD4 T-cell count at switch, cells $/ \mathrm{mm}^{3}($ mean $\pm \mathrm{SD})$ & $677.86 \pm 329.15$ \\
\hline $\begin{array}{l}\text { Treatment (n,\%) } \\
\text { - Triple therapy } \\
\text { oTDF/FTC } \\
\text { oABC/3TC } \\
\text { - Dual therapy } \\
\text { - Monotherapy }\end{array}$ & $\begin{array}{l}78(52 \%) \\
31(39.7 \%) \\
43(55.1 \%) \\
54(36 \%) \\
18(12 \%)\end{array}$ \\
\hline $\mathrm{TDF}(\mathrm{n}, \%)$ & $37(24.7 \%)$ \\
\hline $\begin{array}{l}\text { Creatinine levels, } \mathrm{mg} / \mathrm{dL}(\text { mean } \pm \mathrm{DS}) \\
\text { eGFR } \mathrm{ml} / \mathrm{min} / 1.73 \mathrm{~m} 2(\text { mean } \pm \mathrm{DS}) \\
-<50 \mathrm{ml} / \mathrm{min} / 1.73 \mathrm{~m} 2(\mathrm{n}, \%) \\
-\quad 50-70 \mathrm{ml} / \mathrm{min} / 1.73 \mathrm{~m} 2(\mathrm{n}, \%) \\
->70 \mathrm{ml} / \mathrm{min} / 1.73 \mathrm{~m} 2(\mathrm{n}, \%)\end{array}$ & $\begin{array}{c}0.92 \pm 0.55 \\
92.60 \pm 17.6 \\
-4(2.66 \%) \\
-6(4 \%) \\
-140(93.33 \%)\end{array}$ \\
\hline $\begin{array}{l}\mathrm{VL}(\mathrm{n}, \%) \\
\text { - Undetectable } \\
-20-50 \text { copies } / \mathrm{mL}\end{array}$ & $\begin{array}{c}136(86.66 \%) \\
14(9.33 \%)\end{array}$ \\
\hline
\end{tabular}

MSM: men who have sex with men; BSX: bisexual; HTX: heterosexual; IDU: injection drugs users; TDF: tenofovirdisoproxilfumarato; FTC: emtricitabine; ABC: abacavir; 3TC: lamivudine; eGFR: estimated glomerular filtration rate; VL: viral load.

Table 1: Demographic and general baseline characteristics

Out of 150 patients studied, 148 had at least one available control after switching, 142 completed 24 weeks of DRV/COBI with available efficacy and safety data, and 115 patients completed 48 weeks with these data available. The flowchart is shown in the Figure 1. At 48 weeks, 22 (14.6\%) patients had changed their treatment (15 for proven or potential drug-drug interactions, 6 for simplification to STR and one for dyslipidemia), and 9 patients (6\%) were lost to follow-up (Table 2).

\begin{tabular}{|c|c|c|c|}
\hline & $\begin{array}{l}\text { 1st control } \\
(n=148)\end{array}$ & $\begin{array}{c}24 w \\
(n=148)\end{array}$ & $\begin{array}{c}48 w \\
(n=148)\end{array}$ \\
\hline HIV-1 VL $<50$ copies/dL & $139(93.9 \%)$ & $133(93.66 \%)$ & $111(96.5 \%)$ \\
\hline $\begin{array}{c}\text { Viral failure } \\
\text { HIV RNA 50-200 } \\
\text { HIV RNA > 200 }\end{array}$ & $\begin{array}{l}9(6.1 \%) \\
\quad 6(66,7 \%)^{1} \\
\quad 3(33,3 \%)^{2}\end{array}$ & $\begin{array}{l}9(6.33 \%) \\
\quad 6(66.6 \%)^{3} \\
\quad 3(33.3 \%)^{4}\end{array}$ & $\begin{array}{l}4(3.5 \%) \\
2(50 \%)^{5} \\
2(50 \%)^{6}\end{array}$ \\
\hline Available virological data & & $142(96 \%)$ & $115(81 \%)$ \\
\hline No virological data & 0 & $6(4 \%)$ & $27(19 \%)$ \\
\hline $\begin{array}{l}\text { Discontinuation for drug-drug interactions with } \\
\text { last available HIV-1 RNA }<50\end{array}$ & & $1(16,7 \%)$ & $14(51.8 \%)$ \\
\hline $\begin{array}{l}\text { Discontinuation for AE with last available HIV-1 } \\
\qquad \text { RNA }<50\end{array}$ & & & $1(3.7 \%)$ \\
\hline $\begin{array}{c}\text { Change in ARV regimen for other reason with last } \\
\text { available HIV-1 RNA }<50\end{array}$ & & & $6(22.2 \%)$ \\
\hline Death & & $1(16,7 \%)$ & $1(3.7 \%)$ \\
\hline Lost & & $4(66,6 \%)$ & $5(18.5 \%)$ \\
\hline
\end{tabular}

${ }^{1}$ Four patients were undetectable in the following control, 2 patients discontinued treatment. ${ }^{2}$ One patient VF while receiving chemotherapy for lymphoma, and was undetectable in the following control; 2 patients discontinued treatment. ${ }^{3}$ Four patients were undetectable in the following control, 1 patient change ARV treatment and 1 patient lost follow-up. ${ }^{4}$ One patient was rescued with the same regimen and was undetectable at the next control, 1 patient died for lung cancer, 1 patient lost follow-up. ${ }^{5}$ One blip and one patient lost follow-up. ${ }^{6}$ Both patients were lost follow-up in further controls. Table 2: Virological outcome after switching to DRV/c 


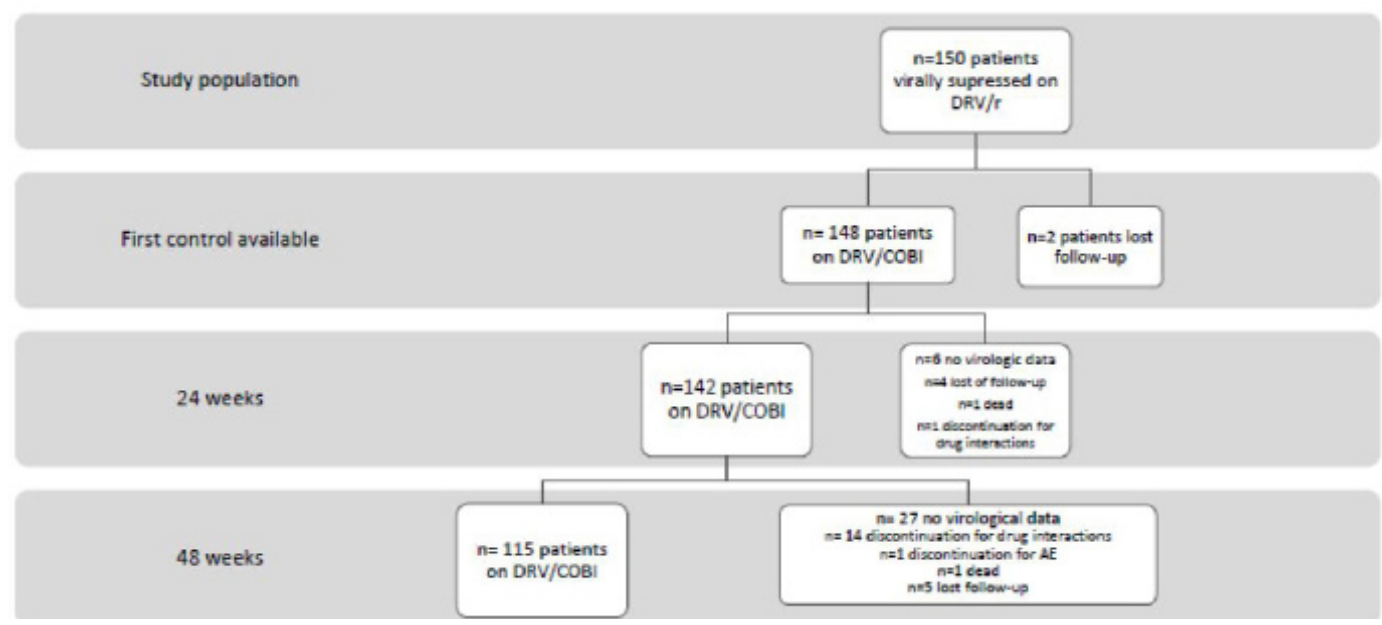

Figure 1: Flowchart study population

\section{Viral Efficacy}

Switching to DRV/COBI maintained viral suppression in $97.2 \%$ of the patients at 48 weeks. In patients who discontinued DRV/ COBI between weeks 24 th and 48th, viral suppression was maintained in $92.9 \%$. Most cases of detectable viremia were "blips", (VL $<200$ copies/dL and $<50$ in the next available control).

At week $48,96 \%$ of patients on triple therapy, $100 \%$ of patients on dual therapy and $92.3 \%$ patients on monotherapy showed $\mathrm{VL}<50 \mathrm{c} / \mathrm{dL}$, with no differences between these three groups. No statistically significant differences were observed in mean CD4 cell counts $(\mathrm{p}=0.19)$.

\section{Safety}

There were no complaints reported by patients after switching from DRV/r to DRV/COBI.

Serum creatinine levels did not change significantly after switching or in the following controls. However, the eGFR was significantly lower after switching in the first available control, then remaining stable at weeks 24 and 48 (Table 3). Performing the analysis in patients receiving TDF and those who did not, the same results were obtained. The initial decrease in eGFR remained significant after the first control in patients with baseline eGFR $>70 \mathrm{ml} / \mathrm{min} / 1.73 \mathrm{~m}^{2}$, with a mean decrease of $6.19 \mathrm{ml} / \mathrm{min} / 1.73 \mathrm{~m}^{2}$ (IC95\% 4.82-7.56, $\mathrm{p}<0.0001)$, and in those with baseline eGFR 50-70 $\mathrm{ml} / \mathrm{min}$, with a mean decrease of $3.5 \mathrm{ml} / \mathrm{min}$, (95\% CI $-0.69-7.69$, $\mathrm{p}=0.044)$.

\begin{tabular}{|l|c|c|c|c|c|c|c|}
\hline & Baseline & $\begin{array}{c}\mathbf{1 s t} \text { control } \\
(\mathbf{1 7 . 4 w )}\end{array}$ & $\mathbf{p}$ & $\mathbf{2 4 w}$ & $\mathbf{p}$ & $\mathbf{4 8 w}$ & $\mathbf{p}$ \\
\hline Creatinine levels, mg/dL (mean \pm DS) & $0.92 \pm 0.55$ & $1.01 \pm 0.59$ & 1 & $0.98 \pm 0.26$ & $1^{1}$ & $0.94 \pm 0.22$ & $1^{1}$ \\
& & & & & & & \\
• On TDF & $0.88 \pm 0.1$ & $0.98 \pm 0.18$ & 1 & $0.98 \pm 0.19$ & $1^{1}$ & $0.97 \pm 0.19$ & $1^{1}$ \\
• Not TDF & $0.93 \pm 0.6$ & $1.01 \pm 0.6$ & 1 & $0.97 \pm 0.3$ & $1^{1}$ & $0.93 \pm 0.3$ & $1^{1}$ \\
\hline eGFR ml/min (mean \pm DS) & $92.60 \pm 17.6$ & $86.51 \pm 18.5$ & $<0.0001$ & $86.24 \pm 17.2$ & $1^{1}$ & $88.83 \pm 165.4$ & 11 \\
• On TDF & $95.23 \pm 11.8$ & $87.71 \pm 12.1$ & $<0.0001$ & $87.78 \pm 12.2$ & $1^{1}$ & $88.41 \pm 16.4$ & $1^{1}$ \\
• Not TDF & $91.6 \pm 19.2$ & $86.1 \pm 19.8$ & $<0.0001$ & $85.75 \pm 18.5$ & $1^{1}$ & $88.91 \pm 16.7$ & $1^{1}$ \\
\hline Total cholesterol, mg/dL (mean \pm DS) & $194.76 \pm 43.1$ & $195.98 \pm 43.3$ & 0.58 & $197.57 \pm 43$ & $0.76^{2}$ & $201.41 \pm 47.3$ & $0.91^{2}$ \\
\hline HDL-Cholesterol, mg/dL(mean \pm DS) & $51.76 \pm 15.7$ & $51 \pm 13.4$ & 0.46 & $50.60 \pm 13.9$ & $0.4^{2}$ & $54.86 \pm 15.4$ & $0.95^{2}$ \\
\hline LDL-Cholesterol, mg/dL (mean \pm DS) & $109.76 \pm 33.9$ & $115.32 \pm 34.6$ & 0.99 & $114.58 \pm 34.3$ & $0.92^{2}$ & $113.18 \pm 35.9$ & $0.52^{2}$ \\
\hline Triglycerides, mg/dL (mean \pm DS) & $168.93 \pm 122.5$ & $159.70 \pm 147.7$ & 0.02 & $172.82 \pm 170.3$ & $0.49^{2}$ & $154.31 \pm 207.5$ & $0.1^{2}$ \\
\hline
\end{tabular}

${ }^{1}$ refers to comparison with the previous control. ${ }^{2}$ refers to comparison with baseline

Table 3: Renal function and lipid profile before and after switch to DRV/c

The lipid profile remained stable after switching (table 3$)$. Only a light decrease in the triglyceride levels $(10 \mathrm{mg} / \mathrm{dL})$ was observed in the first control after switching $(\mathrm{p}=0.02)$.

\section{Discussion}

RTV has been the only available PI boosting agent in the last 15 years, although its complex spectrum of drug-drug interactions and unfavorable metabolic effects make it difficult to use in some patients. COBI is a new booster that allows to improve the dosage of several antiretroviral drugs, with potentially lower pharmacokinetic interactions, and whose molecular characteristics allows its co-formulation with other drugs in a single pill, thus favoring optimal adherence to ART, essential for long- term effectiveness. 
In our experience, the direct change from DRV/r to a DRV/COBI in a single pill maintains viral suppression, and is safe in clinical practice. Patients who failed did so because of a recognized lack of adherence or serious clinical situations leading to discontinuation (abdominal sepsis in one patient, and mucositis by chemotherapy), with no evidence of emerging resistance mutations.

Evidence on viral efficacy and safety of direct switching DRV/r to DRV/COBI in suppressed patients is currently scarce. Atazanavir/ COBI showed non-inferior to atazanavir/RTV in a randomized, double-blinded clinical trial at 48 weeks in naïve patients [9]. In an open, single-arm clinical trial also in naïve patients [12], DRV/COBI once daily was well tolerated, with no new safety concerns. Pharmacokinetics, viral and immunologic responses for DRV/COBI were similar to previous data available for DRV/r. However, to our knowledge, this is the first study reporting on the effect of this simplification strategy in clinical practice.

In our series there were no relevant side effects leading to treatment withdrawal. Inhibition of CYP3A4 with RTV is non-selective and may interfere with other drug metabolism pathways, resulting in a high potential for pharmacological interactions. The design of this study does not allow us to draw conclusions about any lower potential for pharmacological interactions attributed to COBI [5], as all patients were treated with DRV/r before. In the 48-week observation period, 15 patients had to change DRV/ $\mathrm{COBI}$ because proven or potential drug-drug interactions, most of them with direct-acting antivirals for hepatitis $\mathrm{C}$ or other comorbidities. There were also no changes in the rate of gastrointestinal adverse effects, probably because a long time on DRV/r had selected a population with no relevant side effects of RTV.

COBI inhibits the renal transporter of creatinine MATE1. Although creatinine is freely filtered into the glomerulus, between 10 and $15 \%$ is actively secreted into the proximal tubule. Inhibition of this tubular secretion results in a slight increase in serum creatinine concentration (10-15\%) and therefore mild reductions in eGFR estimated by the usual formulas. However, current GFR measured by other methods is not modified by COBI [13]. Several clinical trials [9-11] have confirmed creatinine elevations of $10-15 \%$ in the first 4 weeks in patients receiving COBI, later remaining stable up to week 96 after reaching a new set point. Consistent with these findings, a small increase in serum creatinine was seen in our study. These changes were observed in the first available control, without further increase through 48 weeks. We observed an increase in serum creatinine of $0.09 \mathrm{mg} / \mathrm{dL}$ (9.78\%), consistent with levels previously reported numbers. Current GFR was not measured in our patients by direct methods. However, since the increments in creatinine observed in our study are within the expected by the mechanism of action of the drug, and based on the absence of greater deterioration of renal function over time, our data seem to confirm an only "cosmetic" effect of COBI on renal function, as observed other studies.

Most previous studies have included patients with eGFR $>70 \mathrm{ml} / \mathrm{min}$. Because full-dose FTC and TDF can be used in patients with $\mathrm{CrCl} \geq 50 \mathrm{~mL} / \mathrm{min}$, COBI was evaluated in patients with mild to moderate renal impairment (50-89 $\mathrm{mL} / \mathrm{min})$ [14], showing the same safety and efficacy. Small reductions in eGFR were observed as early as by week 2, after which they remained stable. Interestingly in this study, changes in eGFR were higher in patients with the higher eGFR $\left(>70 \mathrm{ml} / \mathrm{min} / \mathrm{m}^{2}\right)$. Our results support previous observations, although the number of patients with eGFR $<70 \mathrm{ml} / \mathrm{min} / \mathrm{m}^{2}$ included in our study was very low.

Of particular interest is the potential cumulative renal effect of COBI in patients on treatment with TDF, particularly if we consider that COBI inhibits intestinal P-glycoprotein, thus increasing the absorption of atazanavir and DRV as well as TDF. Some observational studies found that boosted PI regimens used with TDF may be associated with a higher incidence of chronic kidney disease or greater decreases in the eGFR $[15,16]$. When regimens containing both COBI and TDF were evaluated $[10,11,17]$, a very low rate of discontinuation by renal cause was observed, none with evidence of proximal tubulopathy. However, the population included in these clinical trials had a low risk of renal failure and, despite the low number of patients who developed tubular disease requiring drug discontinuation; clinicians should be alert to the nephrotoxic potential of the combination of COBI with other nephrotoxic drugs.

In our series, a lower increase in creatinine levels is observed in patients receiving TDF at week 48 than in those who do not, a finding that would support the renal safety of the combination. In our opinion, a possible explanation could be that patients on TDF already have a somewhat inhibited tubular creatinine secretion, although the analysis performed in this study does not permit to conclude this hypothesis. Further studies would be needed to clarify this possible mechanism.

One of the main drawbacks of prolonged treatment with RTV is its unfavorable metabolic effect, including lipodystrophy, dyslipidemia and insulin resistance. Studies with COBI in healthy volunteers suggest a lower negative impact on lipid metabolism. However, in the previously mentioned study by Gallant et al. [12], naïve patients receiving ATV/RTV or ATV/COBI did not show significant differences in plasma lipid levels from baseline in either group at 48 weeks. In our study, levels of total cholesterol, LDL cholesterol and HDL cholesterol remained stable, while triglyceride levels showed a mild decrease, which reached statistical significance, supporting the lower metabolic impact of COBI, but of minor clinical significance. Long-term studies assessing the metabolic profile of cobicistat would be also needed.

Our study presents a series of limitations that make it difficult to extrapolate results. First, it is a retrospective review of clinical practice, with the limitations inherent to this type of studies. Second, the analytical evaluation after switching took place after a variable time interval. Third, this is a short-term study, which probably does not allow a correct assessment of changes in the lipid 
profile. By the contrary, the main contributions of our series are the analysis of a considerable number of patients in actual clinical practice, who were consecutively switched without any selection bias, and followed trough a year.

\section{Conclusion}

In conclusion, $\mathrm{COBI}$ is an alternative to ritonavir as a booster of antiretroviral drugs, including PIs, allowing their co-formulation in a single tablet. In HIV-1-infected, virally suppressed patients, switching from DRV/r to DRV/ is safe and effective in maintaining viral suppression through 48 weeks. The renal safety profile of COBI in this study was consistent with the long-term from phase 3 studies of COBI-containing regimens. The mild and non-progressive elevation of serum creatinine, which appears to be lower in patients treated with baseline eGFR below $70 \mathrm{ml} / \mathrm{min}$, is confirmed. DRV/c does not have a relevant effect on the lipid profile, at least immediately.

\section{References}

1. Hsu A, Granneman GR, Bertz RJ (1998) Ritonavir. Clinical pharmacokinetics and interactions with other anti-HIV agents. Clin Pharmacokinet 35: 275-91.

2. Orkin C, DeJesus E, Khanlou H, Stoehr A, Supparatpinyo K, et al. (2013) Final 192-week efficacy and safety of once-daily darunavir/ritonavir compared with lopinavir/ritonavir in HIV-1-infected treatment-naïve patients in the ARTEMIS trial. HIV Med 14: 49-59.

3. Cahn P, Fourie J, Grinsztejn B, Hodder S, Molina JM, et al. (2011) Week 48 analysis of once-daily vs. twice-daily darunavir/ritonavir in treatment-experienced HIV-1-infected patients. AIDS 25: 929-39.

4. Mathias AA, German P, Murray BP, Wei L, Jain A, et al. (2010) Pharmacokinetics and pharmacodynamics of GS-9350: a novel pharmacokinetic enhancer without anti-HIV activity. Clin Pharmacol Ther 87: 322-29.

5. Xu L, Liu H, Murray B, Callebaut C, Lee MS, et al. (2010) Cobicistat (GS-9350): a potent and selective inhibitor of human CYP3A as a novel pharmacoenhancer. ACS Med ChemLett 1: 209-13.

6. Temesgen Z (2013) Cobicistat, a pharmacoenhancer for HIV treatments. Drugs Today (Barc) 49: 233-7.

7. Kakuda TN, Opsomer M, Timmers M, Iterbeke K, Van De Casteele T, et al. (2014) Pharmacokinetics of darunavir in fixed-dose combination with cobicistat compared with coadministration of darunavir and ritonavir as single agents in healthy volunteers. J Clin Pharmacol 54: 949-57.

8. European Medicines Agency (2014) Committee for Human Products for Medicinal Use. Summary of opinion: Rezolsta (darunavir/cobicistat). 25 September 2014. Accessed on September 20, 2017.

9. Gallant JE, Koenig E, Andrade-Villanueva J, Chetchotisakd P, DeJesus E, et al. (2013) Cobicistat versus ritonavir as a pharmacoenhancer of atazanavir plus emtricitabine/tenofovirdisoproxilfumarate in treatment-naive HIV type 1-infected patients: week 48 results. J Infect Dis 208: $32-9$.

10. Sax PE, DeJesus E, Mills A, Zolopa A, Cohen C, et al. (2012) Co-formulated elvitegravir, cobicistat, emtricitabine, and tenofovir versus co-formulated efavirenz, emtricitabine, and tenofovir for initial treatment of HIV-1 infection: a randomised, double-blind, phase 3 trial, analysis of results after 48 weeks. Lancet 379: 2439 48.

11. DeJesus E, Rockstroh JK, Henry K, Molina JM, Gathe J, et al. (2012) Co-formulated elvitegravir, cobicistat, emtricitabine, and tenofovirdisoproxilfumarate versus ritonavir-boosted atazanavir plus co-formulated emtricitabine and tenofovirdisoproxilfumarate for initial treatment of HIV-1 infection: a randomised, double-blind, phase 3, non-inferiority trial. Lancet 379:2429-38.

12. Tashima K, Crofoot G, Tomaka FL, Kakuda TN, Brochot A, et al. (2014) Cobicistat-boosted darunavir in HIV-1-infected adults: week 48 results of a Phase IIIb, open-label single-arm trial. AIDS Res Ther 11: 39-44.

13. German P, Liu HC, Szwarcberg J, Hepner M, Andrews J, et al. (2012) Effect of cobicistat on glomerular filtration rate in subjects with normal and impaired renal function. J Acquir Immune DeficSyndr 61: 32-40

14. McDonald CK, Fisher M, Custodio J, Martorell C, Post FA, et al. (2014) Cobicistat-Boosted Protease Inhibitors in HIV-Infected Patients with Mild to Moderate Renal Impairment. HIV Clin Trials 15: 269-73.

15. Mocroft A, Kirk O, Reiss P, De Wit S, Sedlacek D, et al. (2010) Estimated glomerular filtration rate, chronic kidney disease and antiretroviral drug use in HIVpositive patients. AIDS 24: 1667-78.

16. Young J, Schafer J, Fux CA, Furrer H, Bernasconi E, et al. (2012) Renal function in patients with HIV starting therapy with tenofovir and either efavirenz, lopinavir or atazanavir. AIDS 26: 567-5.

17. Stray KM, Bam RA, Birkus G, Hao J, Lepist EI, et al. (2013) Evaluation of the effect of cobicistat on the in vitro renal transport and cytotoxicity potential of tenofovir. Antimicrob Agents Chemother 57: 4982-9. 


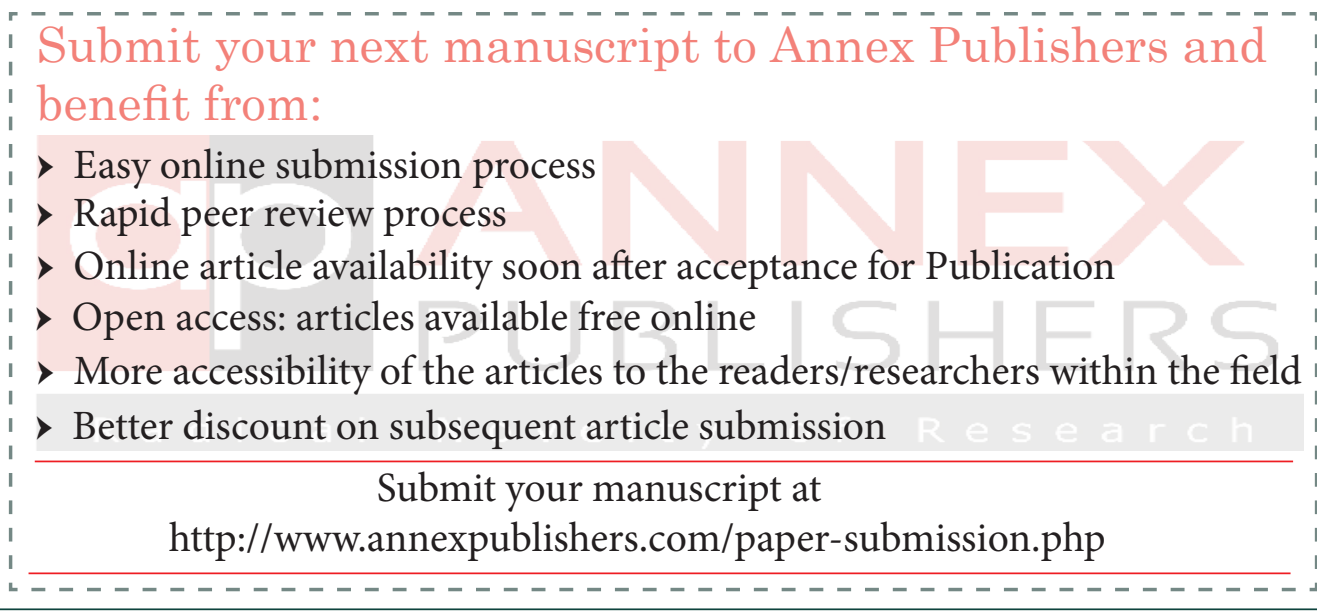

